\title{
Fractional kinetics of photocatalytic degradation
}

\author{
C. L. Wang \\ School of Physics, Shandong University \\ Jinan, Shandong 250100, P. R. China \\ wangcl@sdu.edu.cn
}

Received 19 August 2018; Accepted 21 September 2018; Published 12 October 2018

\begin{abstract}
In this paper, photocatalytic degradation processes of different materials are fitted to the first-order kinetic model, second-order kinetic model and fractional first-order kinetic model. Deterministic coefficients are calculated for the evaluation of the validity of these models. The fitting results show clearly that the degradation process can fit the fractional first-order kinetic model in a very good manner. In this way, two material parameters can be well defined. One is the degradation time, which can be used to describe the photocatalytic degradation process quantitatively. Another is the order of the derivative, which could be related to the material's microstructure.
\end{abstract}

Keywords: Chemical reaction kinetics; photocatalytic materials; fractional calculus.

\section{Introduction}

Universal dielectric relaxation law is a milestone in understanding the dielectric behavior of materials. ${ }^{1}$ Since it has been proposed by Jonscher after analysis of numerous experimental data in his late years, much progress has been made, e.g., by Stanislavsky and Weron, ${ }^{2}$ Nigmatullin et al. ${ }^{3}$ and Garrappa et al. ${ }^{4}$ Now we know that the fractional kinetics belongs to the domain of influence of universal relaxation law. ${ }^{5}$ Though it has been found originally in dielectric relaxations, the kind of universality can be found in many other properties of materials, e.g., luminescence and photoconductivity, chemical reaction kinetics as well as mechanical relaxation. ${ }^{6}$ In this paper, we show that the photocatalytic degradation process can be well described by the fractional differential equation model.

Degradation under irradiation is a standard approach for developing the activity of photocatalytic materials. The degradation process is normally characterized by chemical reaction kinetic equations. In practice, the photocatalytic degradation has been found to be a pseudo-first-order kinetic model, since it does not follow the first-order kinetic equations exactly. Few examples are the methyl orange dye decomposition over $\mathrm{Ag} @ \mathrm{AgCl}$ catalyst under irradiation of visible light, ${ }^{7}$ decolorization of $\mathrm{RhB}$ aqueous solution with hierarchical flower-like tungsten trioxide assemblies ${ }^{8}$ and decolorization of methyl orange over porous $\mathrm{AgBr} / \mathrm{Ag}$ composite microspheres under visible-light irradiation. ${ }^{9}$ However, it has been found in a recent work that the degradation process in $\mathrm{Ag} / \mathrm{AgBr}$-based system can be much better described by pseudo-second-order kinetic reaction model, rather than the pseudo-first-order kinetic model. ${ }^{10}$ On the other hand, kinetic process of fractional first-order kinetics has been observed recently on radioactive decay process of polyfluorene. ${ }^{11}$ To eliminate the controversy on the kinetic order of photocatalytic degradation, the experimental data are fitted with first-order, second-order and fractional first-order kinetic models with the least-squares method. Coefficient of determination is calculated for the quantitative evaluation of the fitting degree of kinetic equation with the experimental data.

\section{Models for Photocatalytic Degradation}

For a first-order kinetic model, the rate of change of concentration is assumed to be proportional to the concentration at a particular instant. Hence the differential equation for a first-order photocatalytic degradation with concentration $C(t)$ at time $t$ is expressed as

$$
\frac{d C(t)}{d t}=-k_{1} C(t)
$$

where $k_{1}$ is called rate constant, ${ }^{8,12}$ with the dimension of inverse of time, normally as $\mathrm{s}^{-1}$. The initial condition is

$$
\left.C(t)\right|_{t=0}=C_{0} .
$$

Here $C_{0}$ is the concentration at zero time, normally the time at which irradiation begins. The solution of differential equation (1) with the above initial condition can be rewritten as

$$
C(t)=C_{0} \cdot e^{-k_{1} t} .
$$

For a second-order kinetic model, the concentration change rate is proportional to the square of the concentration at the particular instant, i.e., the differential equation for a secondorder photocatalytic degradation is

$$
\frac{d C(t)}{d t}=-k_{2} C^{2}(t)
$$

This is an Open Access article published by World Scientific Publishing Company. It is distributed under the terms of the Creative Commons Attribution 4.0 (CC-BY) License. Further distribution of this work is permitted, provided the original work is properly cited. 
where $k_{2}$ is also called as rate constant, ${ }^{10,13}$ with its dimension as $\mathrm{cm}^{3} /$ mole.s. The initial condition is same as Eq. (2). The solution is normally expressed as

$$
\frac{1}{C_{0}}-\frac{1}{C(t)}=k_{2} t
$$

For a fractional first-order kinetic model the differential equation is similar to the first-order kinetic model, except that the derivative of concentration with respect to time is not an integer, but a real number. The differential equation for a fractional first-order kinetic model is ${ }^{11}$

$$
{ }_{0}^{C} D_{t}^{\alpha}[C(t)]=-\frac{C(t)}{\tau^{\alpha}},
$$

where the derivative is of $\alpha$ order under Caputo sense, i.e.,

$$
{ }_{0}^{C} D_{t}^{\alpha}[C(t)]=\frac{1}{\Gamma(1-\alpha)} \int_{0}^{t} \frac{1}{(t-x)^{\alpha}}\left[\frac{d}{d t} C(x)\right] d x,
$$

where $\tau$ is the characteristic degradation time. The initial condition is same as Eq. (2). Solution of differential equation (6) is

$$
C(t)=C_{0} \cdot E_{\alpha}\left(-\left(\frac{t}{\tau}\right)^{\alpha}\right)
$$

where $E_{\alpha}$ is a single-parameter Mittag-Leffler function, ${ }^{14}$ defined as

$$
E_{\alpha}(z)=\sum_{k=0}^{\infty} \frac{z^{k}}{\Gamma(\alpha k+1)},
$$

where $\Gamma(\cdot)$ is the Gamma function. When $\alpha=1$, the singleparameter Mittag-Leffler function retreats to an exponential function, i.e., fractional first-order kinetic model reduces to a first-order kinetic model.

\section{Fitting Method}

The least-squares method is adopted to fit the above three kinetic models with experimental data. For the first-order and second-order kinetic models, linear regression can be easily applied when the variables are redefined. For the first-order kinetic model, Eq. (3) can be rewritten as

$$
\log \left[C(t) / C_{0}\right]=-\frac{t}{\tau_{1}}
$$

where $\tau_{1}=1 / k_{1}$ is defined as the degradation time of firstorder kinetic model. Above equation can be used for direct linear regression fitting when we take the logarithmic term as the fitting function. For the second-order kinetic model, Eq. (5) can be rewritten as

$$
\frac{C_{0}}{C(t)}-1=-\frac{t}{\tau_{2}},
$$

where $\tau_{2}=1 / C_{0} k_{2}$ is defined as the degradation time of second-order kinetic model. Also, the linear regression fitting can be applied directly when we take the left-hand term of above equation as the fitting function.
The values of degradation times $\tau_{1}$ and $\tau_{2}$ will be returned by fitting the experimental data with Eqs. (10) and (11). By introducing the degradation time, we can have a direct comparison of the fitting results with different kinetic models, since rate constant $k_{1}$ of the first-order kinetic model has a different dimension from $k_{2}$ of the second-order kinetic model.

For fractional first-order kinetic model, a Monte Carlo method is used to fit Eq. (8) with the experimental data. An initial trial solution is adopted from the fitting value of the first-order kinetic model, i.e., we start with $\alpha=1$, and $\tau=\tau_{1}$ from fitting with Eq. (10) as the initial trial. Then a new pair of trial solution $\left(\alpha_{\text {trial }}, \tau_{\text {trial }}\right)$ around the initial trials is generated as

$$
\alpha_{\text {trial }}=\alpha \cdot(0.5-\text { rand }), \quad \tau_{\text {trial }}=\tau \cdot(0.5-\text { rand }),
$$

where rand is a random number between 1 and 0 . When the new trial pair of parameters produces a less tolerance, we keep this pair of parameters as an updated pair of solution $(\alpha, \tau)$, otherwise we discard them. Another pair of trial solution will be generated from the updated pair of solution as in Eq. (12), then the tolerance is calculated. This procedure is repeated until an expected tolerance is reached, and parameters $\alpha$ and $\tau$ are returned simultaneously for the fractional first-order kinetic model.

Coefficient of determination is a statistical measure of how well the regression line approximates the real data points. ${ }^{15}$ The definition of the coefficient of determination $R^{2}$ is

$$
R^{2} \equiv 1-\frac{\mathrm{SS}_{\mathrm{res}}}{\mathrm{SS}_{\mathrm{tot}}}
$$

where $\mathrm{SS}_{\text {tot }}$ is the total sum of squares and $\mathrm{SS}_{\text {res }}$ is the sum of squares of residuals, respectively, i.e.,

$$
\mathrm{SS}_{\mathrm{tot}}=\sum_{i=1}^{n}\left(y_{i}-\bar{y}\right)^{2}, \quad \mathrm{SS}_{\mathrm{res}}=\sum_{i=1}^{n}\left(y_{i}-f_{i}\right)^{2} \text {. }
$$

Here $n$ is the number of experimental data, $y_{i}$ is $i$ th experimental value, $\bar{y}$ is the mean experimental value and $f_{i}$ stands for $i$ th modeled value. The coefficient of determination normally ranges from 0 to 1 . Value of 1 or 0 indicates that the regression line represents all or none of the data, respectively. A higher value of coefficient is an indicator of a better fit for the observations.

\section{Results and Discussion}

The first example is photocatalytic degradation of RhB solution with tungsten trioxide. The experimental data are taken from work by $\mathrm{Yu}$ and $\mathrm{Qi} .{ }^{8}$ Hierarchical flower-like tungsten trioxide assemblies have been prepared through a simple hydrothermal method. The fitting curves of tungsten trioxide for the photocatalytic decolorization of RhB aqueous solution at ambient temperature are shown in Fig. 1, and the fitting parameters are listed in Table 1. The sample notations W2, 


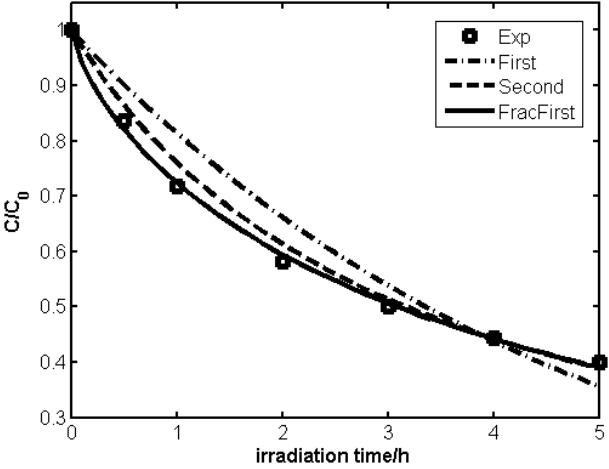

(a)

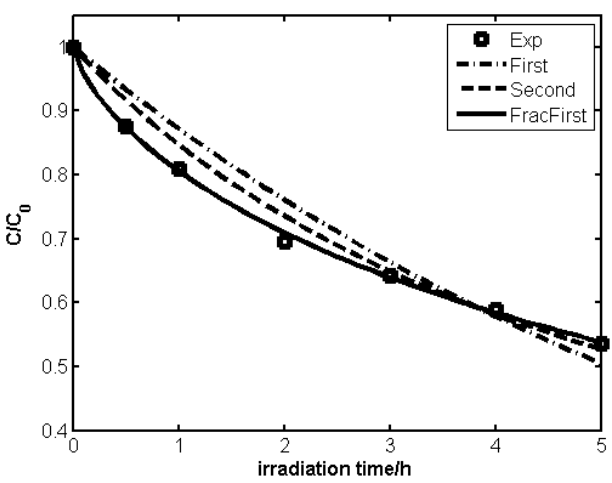

(c)

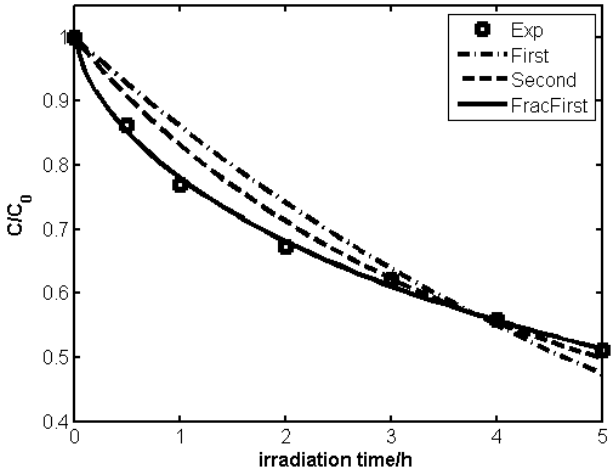

(b)

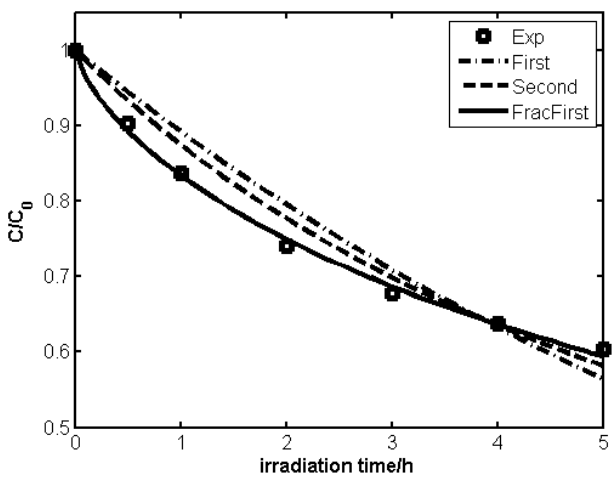

(d)

Fig. 1. Photocatalytic decolorization of RhB aqueous solution with tungsten trioxide. "Exp" denotes experimental data, "First" denotes firstorder kinetic model, "Second" denotes second-order kinetic model and "FracFirst" denotes fractional first-order kinetic model. Degradation percentage ratios for (a) W2, (b) W3, (c) W6 and (d) W7.

W3, W6 and W7 in caption of Fig. 1 and Table 1 are taken from Ref. 8. All these four samples are prepared under the same conditions, but W2 was treated with $0.012 \mathrm{~mol} / \mathrm{L}$ $\mathrm{HNO}_{3}$, W3 with $0.018 \mathrm{~mol} / \mathrm{L} \mathrm{HNO}_{3}$, W6 with $0.006 \mathrm{~mol} / \mathrm{L}$ $\mathrm{HCl}$ and $\mathrm{W} 7$ with $0.012 \mathrm{~mol} / \mathrm{L} \mathrm{H}_{2} \mathrm{SO}_{4}{ }^{8}$ Open squares in Fig. 1 denote the experimental data, dot-dashed lines denote the first-order kinetic model fittings, dashed lines denote the second-order kinetic model fittings and solid lines denote the fractional first-order model fittings.

It can be clearly seen from Fig. 1 that the experimental degradation curve fits the fractional first-order kinetic model much better than the integer-order kinetic processes, i.e., the first-order and the second-order kinetic models. Nearly all the

Table 1. Fitting parameters for photocatalytic degradation of RhB solution with tungsten trioxide. ${ }^{8}$

\begin{tabular}{lcccccccccc}
\hline & \multicolumn{2}{c}{ First-order } & & \multicolumn{2}{c}{ Second-order } & & \multicolumn{3}{c}{ Fractional-order } \\
\cline { 2 - 3 } Sample & $\tau(\mathrm{h})$ & $R^{2}(\%)$ & & $\tau(\mathrm{h})$ & $R^{2}(\%)$ & & $\alpha$ & $\tau(\mathrm{h})$ & $R^{2}(\%)$ \\
\hline W2 & 4.84 & 92.06 & & 3.17 & 98.67 & & 0.73 & 4.90 & 99.79 \\
W3 & 6.70 & 89.72 & & 4.96 & 95.87 & & 0.67 & 8.67 & 99.81 \\
W6 & 7.32 & 92.21 & & 5.55 & 97.08 & & 0.70 & 9.62 & 99.84 \\
W7 & 8.74 & 92.00 & & 6.96 & 96.47 & & 0.69 & 12.84 & 99.72 \\
\hline
\end{tabular}

experimental data lie on the lines of the fractional first-order kinetic model. This suggests that photocatalytic degradation of $\mathrm{RhB}$ solution with tungsten trioxide follows the fractional first-order kinetic model. From Table 1, it can be easily found that the coefficient of determination $R^{2}$ for the fractional firstorder kinetic model is very close to 1 . Values of $R^{2}$ for firstorder and second-order models are smaller than that for fractional first-order kinetic model. The $R^{2}$ values further confirm that the photocatalytic degradation is a fractional first-order kinetic process. Also, the $R^{2}$ values imply that the fitting of second-order kinetic model is better than that of first-order kinetic model, since $R^{2}$ values of second-order kinetic model are larger than those for first-order kinetic model. In all samples, the trend of the degradation times from three kinetic models is the same. Sample W6 has the best photocatalytic performance. From the results in Fig. 1 and Table 1, we can claim that the degradation time from fractional first-order kinetic model can be well defined. The order of derivative $\alpha$ is around 0.7 , which is much lower than 1 for the integer-order kinetic models.

The second example is the photocatalytic degradation of $\mathrm{RhB}$ solution with $\mathrm{Ag} / \mathrm{AgCl} / \mathrm{PrGO}$ (photoreduced graphene oxide) composites. ${ }^{9}$ Silver/silver halide-based nanomaterials have drawn much attention due to their significantly 
improved photocatalytic ability under visible light. Introduction of graphene oxide can obviously enhance the photocatalytic performance of the $\mathrm{Ag} / \mathrm{AgCl}$ composites. The experimental data of photocatalytic degradation of $\mathrm{RhB}$ solution with $\mathrm{Ag} / \mathrm{AgCl} / \mathrm{PrGO}$ composites are taken from the work by Lou et al. ${ }^{9}$ The fitting curves of $\mathrm{Ag} / \mathrm{AgCl} / \mathrm{PrGO}$ composites for the photocatalytic degradation of $\mathrm{RhB}$ are shown in Fig. 2, with fitting parameters being listed in Table 2. The sample notations in caption of Fig. 2 and first column in Table 2 are the same as in Ref. 9.

It can be easily seen from Fig. 2 that all the experimental curves fit perfectly well the fractional first-order kinetics

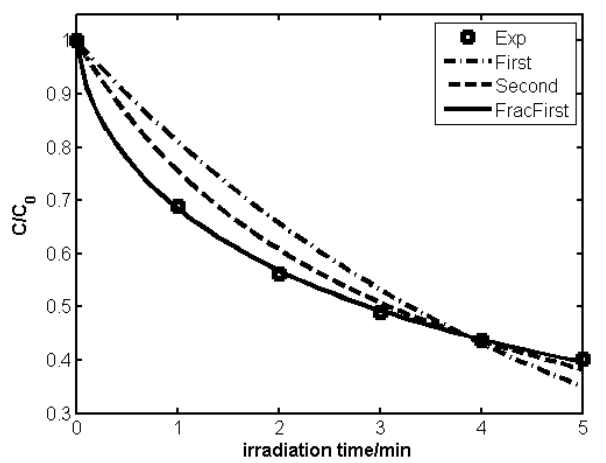

(a)

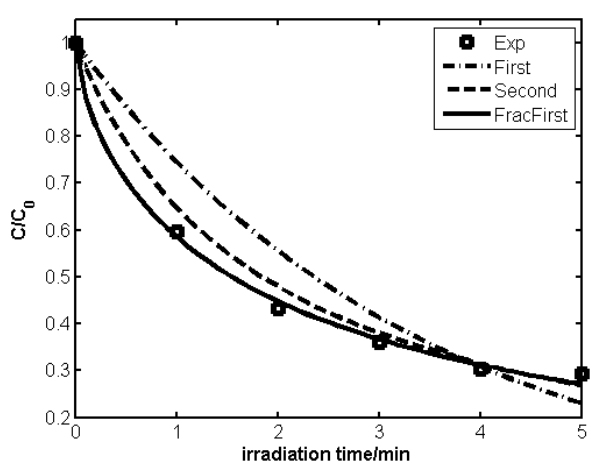

(c)

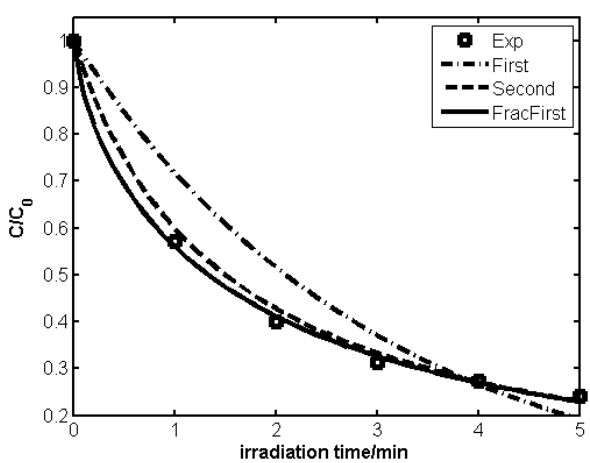

(e) model, much better than that of the integer-order kinetic models. All the experimental data lie nearly on the solid lines, i.e., curves from fractional first-order kinetic model. The coefficient of determination $\left(R^{2}\right)$ values in Table 2 are clearly indicative of the validation of the fractional first-order kinetic model in this degradation process. Exceptions occur in Figs. 2(d) and 2(h) which show that both fractional first-order and second-order kinetic models can fit the experimental data fairly well. The coefficients of determination $R^{2}$ for these two fittings, as listed in Table 2 for fractional first-order and second-order models, are much close to 1. Overall, the fractional first-order kinetic model can fit all the cases

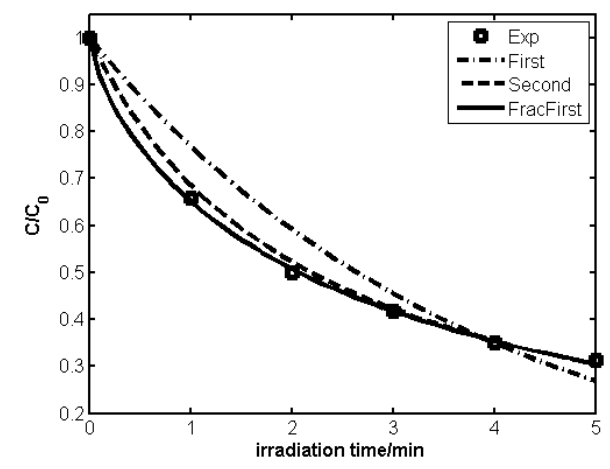

(b)

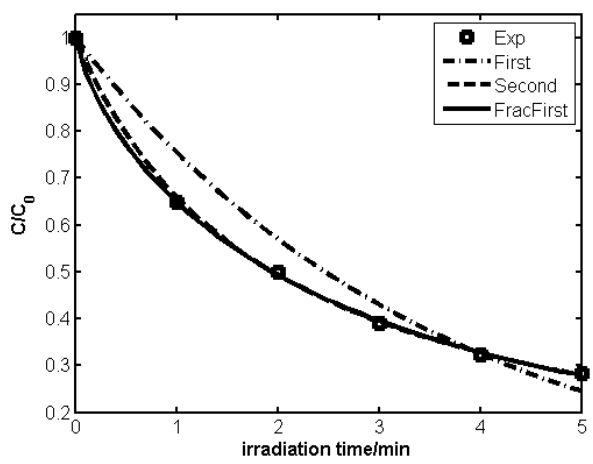

(d)

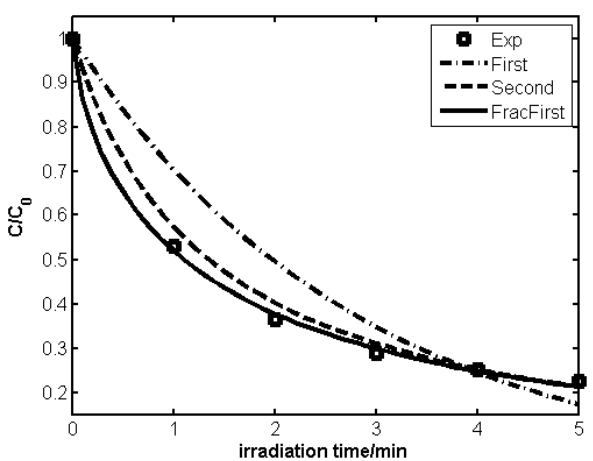

(f)

Fig. 2. Fitting of photocatalytic degradation of $\mathrm{RhB}$ with $\mathrm{Ag} / \mathrm{AgCl} / \mathrm{PrGO}$ composites. ${ }^{9}$ The legend is the same as in Fig. 1. Degradation percentage ratios for (a) Ag/AgBr, (b) Ag/AgBr/PrGO (0 h), (c) Ag/AgBr/PrGO (1 h), (d) Ag/AgBr/PrGO (2 h), (e) Ag/AgBr/PrGO (4h), (f) $\mathrm{Ag} / \mathrm{AgBr} / \mathrm{PrGO}(8 \mathrm{~h}),(\mathrm{g}) \mathrm{Ag} / \mathrm{AgBr} / \mathrm{PrGO}(12 \mathrm{~h})$ and (h) Ag/AgBr/PrGO (16h). 


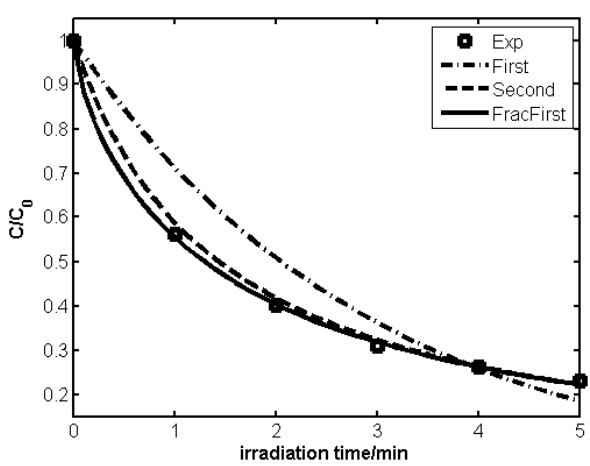

(g)

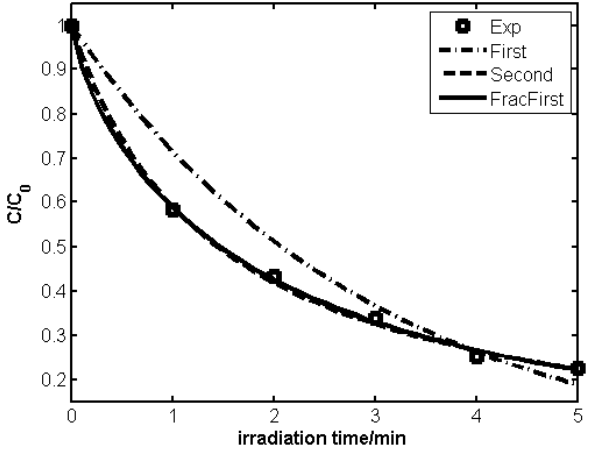

(h)

Fig. 2. (Continued)

globally. The order of derivative $\alpha$ is also around 0.7 , which is much lower than 1 for the integer-order kinetic models.

The third example is the photocatalytic oxidization activity of $\mathrm{NaNbO}_{3}$ single-crystal thin films. ${ }^{16}$ The experimental data are taken from $\mathrm{NaNbO}_{3}$ thin films deposited on $\mathrm{LaAlO}_{3}$ (100), (110) and (111) substrates by pulsed laser deposition technique. Films with (100), (110) and (111) preferentially-oriented crystal planes have been obtained. Their photocatalytic activities have been evaluated by $\mathrm{RhB}$ degradation under the full-arc irradiation of Xeon lamp. ${ }^{16}$ Fitting curves of different crystal planes for the photocatalytic degradation of $\mathrm{RhB}$ are shown in Fig. 3, with fitting parameters being listed in Table 3. The sample notations in Fig. 3 caption and the first column in Table 3 are the same as in Ref. 16. All the fittings are good with the fractional first-order kinetic model. The degradation process in Fig. 3(a) seems far too complete, as it can be expressed as a linear relation between concentration and time. This seems little bit trivial in distinguishing the difference between the three kinds of kinetic model. The degradation process with (100) plane can be fitted well with both second-order kinetic model and fractional first-order kinetic model, as shown in Fig. 3(b). This can be also seen in Table 3 from the coefficients of determination $R^{2}$, which is $99.85 \%$ for second-order kinetic model

Table 2. Fitting parameters of photocatalytic degradation of $\mathrm{RhB}$ with $\mathrm{Ag} / \mathrm{AgCl} /$ PrGO composites. ${ }^{9}$

\begin{tabular}{|c|c|c|c|c|c|c|c|}
\hline \multirow[b]{2}{*}{ Sample } & \multicolumn{2}{|c|}{ First-order } & \multicolumn{2}{|c|}{ Second-order } & \multicolumn{3}{|c|}{ Fractional-order } \\
\hline & $\tau(\min )$ & $R^{2}(\%)$ & $\tau(\min )$ & $R^{2}(\%)$ & $\alpha$ & $\tau(\min )$ & $R^{2}(\%)$ \\
\hline $\mathrm{Ag} / \mathrm{AgBr}$ & 4.76 & 88.61 & 3.10 & 97.10 & 0.64 & 4.72 & 99.95 \\
\hline $\mathrm{Ag} / \mathrm{AgBr} / \mathrm{PrGO}(0 \mathrm{~h})$ & 3.81 & 92.73 & 2.19 & 99.57 & 0.73 & 3.30 & 99.94 \\
\hline $\mathrm{Ag} / \mathrm{AgBr} / \mathrm{PrGO}(1 \mathrm{~h})$ & 3.40 & 87.97 & 1.84 & 98.40 & 0.68 & 2.50 & 99.75 \\
\hline $\mathrm{Ag} / \mathrm{AgBr} / \mathrm{PrGO}(2 \mathrm{~h})$ & 3.56 & 94.68 & 1.95 & 99.95 & 0.77 & 3.06 & 99.98 \\
\hline $\mathrm{Ag} / \mathrm{AgBr} / \mathrm{PrGO}(4 \mathrm{~h})$ & 3.03 & 90.13 & 1.50 & 99.51 & 0.71 & 2.13 & 99.88 \\
\hline $\mathrm{Ag} / \mathrm{AgBr} / \mathrm{PrGO}(8 \mathrm{~h})$ & 2.84 & 87.65 & 1.34 & 99.08 & 0.69 & 1.78 & 99.87 \\
\hline $\mathrm{Ag} / \mathrm{AgBr} / \mathrm{PrGO}(12 \mathrm{~h})$ & 2.95 & 90.60 & 1.43 & 99.69 & 0.72 & 2.06 & 99.95 \\
\hline $\mathrm{Ag} / \mathrm{AgBr} / \mathrm{PrGO}(16 \mathrm{~h})$ & 2.99 & 93.69 & 1.44 & 99.84 & 0.77 & 2.30 & 99.90 \\
\hline
\end{tabular}

and $99.86 \%$ for fractional first-order kinetic model. The degradation process with (111) plane can be fitted well with both first-order kinetic model and fractional first-order kinetic model, as shown in Fig. 3(d). The fractional first-order kinetic model fits the experimental data slightly better than the firstorder kinetic model in this case, as can be seen from the coefficients of determination $R^{2}$. Also the order of derivative of the fractional first-order kinetic model is 0.98 , which is very close to 1 or the order of integer derivative.

From the above fitting results, we can see that the fractional first-order kinetic model can give a unified and quantitative description of the photocatalytic degradation process. Two well-defined model parameters, i.e., degradation time and order of derivative, can be used as material constants for characterizing the photocatalytic behavior. The order of derivative for tungsten trioxide and $\mathrm{Ag} / \mathrm{AgCl} / \mathrm{PrGO}$ composites is around 0.7 , and it is around 0.9 for $\mathrm{NaNbO}_{3}$ thin films. The tungsten trioxide has a hierarchical flower-like structure, and $\mathrm{Ag} / \mathrm{AgCl} / \mathrm{PrGO}$ composites are heterostructured. The microstructures of these two materials are much complicated than that of $\mathrm{NaNbO}_{3}$ thin films. This fact implies that the more complicated the microstructure is, the lower is the order of derivative. However this assertion needs further confirmation from fitting with more experimental data.

Deviation from integer-order kinetic chemical reaction has been noticed many years ago. ${ }^{6,17}$ Kopelman ${ }^{17}$ has noticed as early as 1988 that diffusion-controlled reactions with geometrical constraints, as found in heterogeneous kinetics, might be described by reactions on fractal domains. The hallmarks of "fractal-like" reactions are anomalous reaction orders and time-dependent reaction rate "constants". These anomalies stem from the nonrandomness of the reactant distributions in low dimensions. Jonscher ${ }^{6}$ has also pointed out in his book in 1996 that the chemical kinetics deviated from standard first-order kinetics, but can be regarded as a kind of universal relaxation law in time domain. There is a general trend toward fractional power-law relaxation regardless of the detailed physical and chemical conditions prevailing in the system, and also regardless of whether or not it 


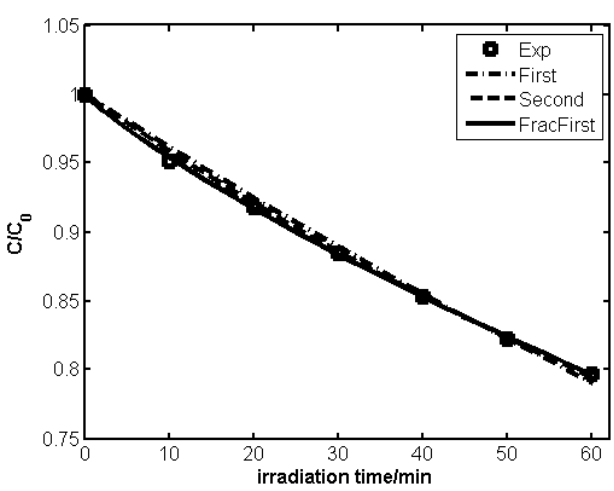

(a)

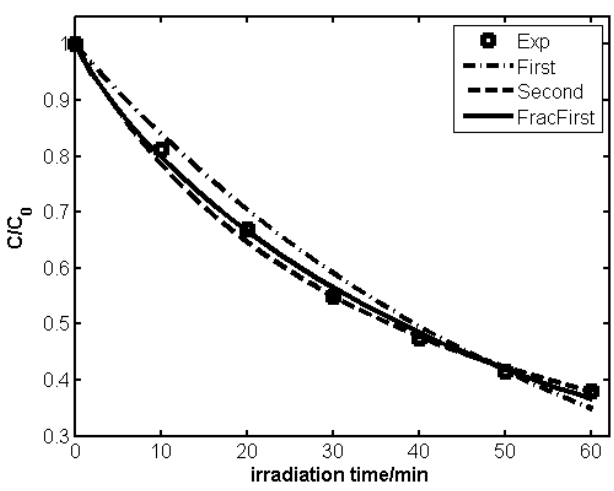

(c)

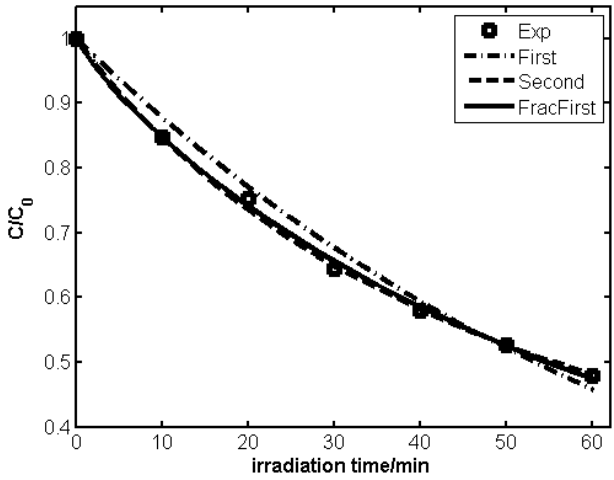

(b)

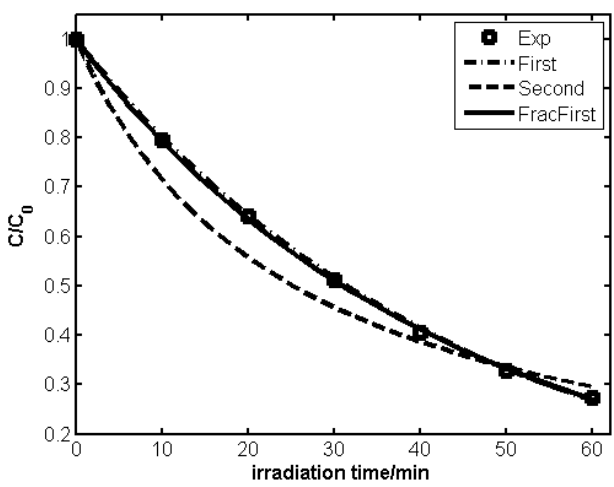

(d)

Fig. 3. Fitting of photocatalytic degradation of $\mathrm{RhB}$ with $\mathrm{NaNbO}_{3} / \mathrm{LaAlO}_{3} \cdot{ }^{16}$ The legend is the same as in Fig. 1 . Degradation percentage ratios for (a) photolysis, (b) plane (100), (c) (110) and (d) (111).

Table 3. Fitting parameters of photocatalytic degradation of $\mathrm{RhB}$ with $\mathrm{NaNbO}_{3} / \mathrm{LaAlO}_{3}$. ${ }^{16}$

\begin{tabular}{|c|c|c|c|c|c|c|c|}
\hline \multirow[b]{2}{*}{ Sample } & \multicolumn{2}{|c|}{ First-order } & \multicolumn{2}{|c|}{ Second-order } & \multicolumn{3}{|c|}{ Fractional-order } \\
\hline & $\tau(\min )$ & $R^{2}(\%)$ & $\tau(\min )$ & $R^{2}(\%)$ & $\alpha$ & $\tau(\min )$ & $R^{2}(\%)$ \\
\hline Photolysis & 255.24 & 99.30 & 232.13 & 99.77 & 0.88 & 332.00 & 99.95 \\
\hline (100) & 76.72 & 98.64 & 55.40 & 99.85 & 0.87 & 82.86 & 99.86 \\
\hline (110) & 56.95 & 98.30 & 36.54 & 99.58 & 0.87 & 57.28 & 99.73 \\
\hline (111) & 45.38 & 99.95 & 25.14 & 95.80 & 0.98 & 44.94 & 99.97 \\
\hline
\end{tabular}

is possible to discern the presence of specific strong interactions or correlations between the various events determining the relaxation regime. ${ }^{6}$ However, to establish the relationship between the order of derivative and the microstructure there is a need for further studies. It is interesting to point out that single-parameter Mittag-Leffler function is also the response function of Cole-Cole dielectric relaxation. ${ }^{6}$ This means that the fractional first-order kinetic equation shares the same differential equation for Cole-Cole relaxation in time domain.

\section{Conclusions}

Experimental data of photocatalytic degradations of different materials have been used for fitting with first-order, second-order and fractional first-order kinetic models. Fitting results have shown clearly that the fractional first-order kinetic model can be applied to all the cases observed experimentally. This implies that the photocatalytic process is of fractional kinetics. The degradation time and order of derivative can be well defined as material constants to characterize their photocatalytic activity. More efforts are needed to fully understand the relationship between the model parameters (i.e., degradation time and order of derivative) and the microstructure of the materials. Also more fitting works have to be done to solidly confirm that photocatalytic degradation has fractional kinetic features.

\section{Acknowledgments}

This work is supported by the National Natural Science Foundation of China (51672159, 51501105 and 51611540342). Author of this work would like to thank Prof. Huang Baibiao of the Institute of Crystal Materials for helping him understand the photocatalysis, Profs. Jiang Xiaoyun and Zhao Weidong of the School of Mathematics, Prof. Li Yan of the School of Control Science of Shandong University, Prof. F. Mainardi of Bologna University of Italy, Prof. R. R. Nigmatullin of Kasan Federal University of Russia, Prof. Igor Podlubny of the Technical University of 
Kosice of Slovak Republic and Prof. Chen Yangquan of the University of California at Merced of USA for helping him understand the fractional calculus.

\section{References}

${ }^{1}$ A. K. Jonscher, Dielectric Relaxation in Solids (Chelsea Dielectrics Press, 1983) [A. K. 琼克, 固体中的介电弛豫 (西安交通大学出版社, 西安, 2008)].

${ }^{2}$ A. Stanislavsky and K. Weron, Stochastic tools hidden behind the empirical dielectric relaxation laws, Rep. Prog. Phys. 80, 036001 (2017).

${ }^{3}$ R. R. Nigmatullin, A. A. Khamzin and D. Baleanu, On the Laplace integral representation of multivariate Mittag-Leffler functions in anomalous relaxation, Math. Methods Appl. Sci. 39, 2983 (2016).

${ }^{4}$ R. Garrappa, F. Mainardi and G. Maione, Models of dielectric relaxation based on completely monotone functions, Fract. Calc. Appl. Anal. 19, 1105 (2016).

${ }^{5}$ V. Uchaikin and R. Sibatov, Fractional Kinetics in Solids (World Scientific, Singapore, 2013).

${ }^{6}$ A. K. Jonscher, Universal Relaxation Law (Chelsea Dielectrics Press, 1996) [A. K. 琼克, 普适弛豫定律体 (西安交通大学出版社, 西安, 2008)].

${ }^{7}$ P. Wang, B. B. Huang, X. Y. Qin, X. Y. Zhang, Y. Dai, J. Y. Wei and M. Whangbo, Ag@ AgCl: A highly efficient and stable photocatalyst active under visible light, Angew. Chem., Int. Ed. 47, 7931 (2008).

${ }^{8}$ J. G. Yu and L. F. Qi, Template-free fabrication of hierarchically flower-like tungsten trioxide assemblies with enhanced visible- light-driven photocatalytic activity, J. Hazard. Mater. 169, 221 (2009).

${ }^{9}$ S. Y. Lou, X. B. Jia, Y. Q. Wang and S. M. Zhou, Templateassisted in-situ synthesis of porous $\mathrm{AgBr} / \mathrm{Ag}$ composite microspheres as highly efficient visible-light photocatalyst, Appl. Catal. B 176-177, 586 (2015).

${ }^{10}$ M. Gao, C. X. Ran, M. Q. Wang, L. Li, Z. W. Sun and X. Yao, The role of reduction extent of graphene oxide in the photocatalytic performance of $\mathrm{Ag} / \mathrm{AgX}(\mathrm{X}=\mathrm{Cl}, \mathrm{Br}) / \mathrm{rGO}$ composites and the pseudo-second-order kinetics reaction nature of the $\mathrm{Ag} / \mathrm{AgBr}$ system, Phys. Chem. Chem. Phys. 18, 18219 (2016).

${ }^{11}$ N. H. T. Lemes, J. P. C. dos Santos and J. P. Braga, A generalized Mittag-Leffler function to describe nonexponential chemical effects, Appl. Math. Model. 40, 7971 (2016).

${ }^{12}$ J. G. Yu, H. G. Yu, B. Cheng, X. J. Zhao, J. C. Yu and W. K. Ho, The effect of calcination temperature on the surface microstructure and photocatalytic activity of $\mathrm{TiO}_{2}$ thin films prepared by liquid phase deposition, J. Phys. Chem. B 107, 13871 (2003).

${ }^{13}$ Y. S. Ho, Review of second-order models for adsorption systems, J. Hazard. Mater. B 136, 681 (2006).

${ }^{14}$ A. Erdélyi (ed.), Higher Transcendental Functions, Vol. 3 (McGraw-Hill, New York, 1955).

${ }^{15}$ Wikipedia, Coefficient of determination (2005), https://en.wikipedia.org/wiki/Coefficient_of_determination.

${ }^{16}$ G. Q. Li, Z. G. Yi, Y. Bai, W. F. Zhang and H. T. Zhang, Anisotropy in photocatalytic oxidization activity of $\mathrm{NaNbO}_{3}$ photocatalyst, Dalton Trans. 41, 10194 (2012).

${ }^{17}$ R. Kopelman, Fractal reaction kinetics, Science 241, 1620 (1988). 